\title{
Eurêka: une intervention tarifaire "à la Tinguely»
}

\section{Urs Stoffel}

Dr méd., membre du Comité central de la FMH, responsable du département Médecine et tarifs ambulatoires

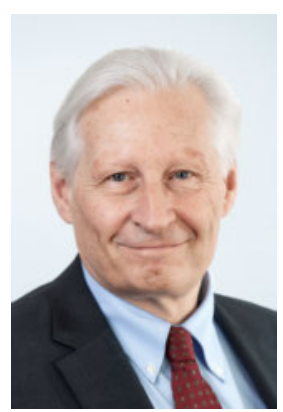

Que se passe-t-il lorsque l'on actionne l'une des roues dentées de la sculpture mondialement célèbre de Jean Tinguely «Eurêka»? Au moins trois autres roues tournent dans l'autre sens.

Pour la seconde fois, le Conseil fédéral veut faire usage de sa compétence subsidiaire au sens de l'art. 43 al. $5^{\text {bis }}$ LAMal et intervenir dans la structure tarifaire ambulatoire à la prestation TARMED, hautement complexe. A la différence de la première intervention en 2014, qui a porté sur un simple transfert de 200 millions au sein du volume du TARMED dans l'intérêt de la médecine de famille et au détriment des disciplines invasives, le Conseil fédéral compte cette fois-ci économiser une somme prétendument «appropriée» de 700 millions de francs.

La deuxième intervention prévue se distingue donc essentiellement de la première, qui a consisté en une redistribution neutre en termes de coûts au moyen d'une réduction linéaire de certaines positions techniques, puisque les mesures actuelles concernent différents niveaux du tarif et visent presque toutes le même objectif: réduire les positions tarifaires!

\section{Catalogue de mesures}

Réduction de minutages de prestations, conversion de prestations à l'acte en prestations au temps, augmentation de la productivité de certaines unités fonctionnelles, suppression des valeurs intrinsèques quantitatives différenciées au profit d'un seul facteur de valeur intrinsèque et nouvelle réduction de $10 \%$ de la plupart des prestations techniques. Et comme si cela ne suffisait pas, de nouvelles règles et restrictions de facturation ont été définies.

\footnotetext{
Voir aussi les graphiques sous www.fmh.ch $\rightarrow$ Tarifs ambulatoires $\rightarrow$ TARMED Tarif $\rightarrow$ Modifications du TARMED à partir du $1^{\mathrm{er}}$ janvier $2018 \rightarrow$ Analyse des mesures.

** Vous trouverez de plus amples informations à l'adresse www.fmh.ch $\rightarrow$ Tarifs ambulatoires $\rightarrow$ Révision du tarif ambulatoire.
}

\section{L'intervention touche toutes les disciplines}

Après avoir analysé et simulé l'impact des mesures sur les différentes disciplines, nous constatons que pratiquement chacune d'entre elles est touchée par la réduction. En raison du cumul et de l'association des mesures, dont certaines portent sur l'ensemble du tarif, plusieurs positions tarifaires enregistrent même des réductions massives et disproportionnées, qui vont à l'encontre de l'économicité et du caractère approprié exigés par la loi.
Nous constatons sans surprise que cela engendre des «effets collatéraux» prévisibles en partie grotesques et involontaires, sous forme de réductions dans des disciplines déjà sous-évaluées dans le tarif actuel, dont la chirurgie pédiatrique, la pédiatrie ou la psychiatrie.

Nous constatons également des transferts considérables au sein même des disciplines: en fonction de leur profil de prestations individuel, certains spécialistes seront fortement touchés par les mesures de réduction, alors que d'autres le seront nettement moins. Dans l'ensemble, l'intervention tarifaire laisse derrière elle un champ de ruines dans la tarification de la médecine ambulatoire en Suisse.* Vous trouverez de plus amples informations à ce sujet dans cette édition du BMS dès la page 512.

\section{Incitations inopportunes au mauvais endroit et au mauvais moment}

Contrairement au principe «l'ambulatoire avant l'hospitalier» soutenu par le Conseil fédéral et le monde politique, cette intervention tarifaire unilatérale, inappropriée et arbitraire génère des incitations inopportunes contraires à une médecine ambulatoire de qualité, efficace et économique. L'intervention affaiblit la prise en charge ambulatoire périphérique avantageuse de la population et va ainsi à l'encontre de la stratégie Santé2020 adoptée par le Conseil fédéral.

\section{Une révision globale appropriée et conforme aux règles applicables en économie d'entreprise est la seule voie à suivre!}

L'ensemble des partenaires tarifaires, y compris la FMH, s'accordent avec le Conseil fédéral pour dire que le TARMED est dépassé et inapproprié et qu'il doit être révisé. Toute nouvelle intervention arbitraire et unilatérale dans le tarif existant ne fait que le déséquilibrer encore davantage. Pour la FMH, une révision tarifaire globale appropriée et conforme aux règles applicables en économie d'entreprise reste la seule voie pertinente à suivre, et c'est donc cette voie que nous allons poursuivre de manière conséquente.** 